\title{
Extracellular vesicles in the black yeast Exophiala dermatitidis: a first characterization
}

Barbara Blasi ${ }^{1}$, Hakim Tafer ${ }^{1}$, Elsa Arcalis ${ }^{2}$, Samyr Kenno ${ }^{3}$, Ulrike Binder ${ }^{3}$, Katja Sterflinger ${ }^{1}$

1 VIBT-Extremophile Center, Department of Biotechnology, University of Natural Resources and Life Sciences,

Muthgasse 18, 1190 Vienna, Austria;

2 Institute for Applied Genetics and Cell Biology, Department of Biotechnology, University of Natural Resources and Life Sciences, Muthgasse 18, 1190 Vienna, Austria;

${ }^{3}$ Division of Hygiene and Medical Microbiology, Medical University Innsbruck, Schöpfstrasse 41, 6020, Innsbruck, Austria.

Introduction. Extracellular vesicles (EVs) are a powerful communication tool in many biological systems and in the last years they have been characterized in several fungi, where they contribute in the transport of molecules across their complex cell wall. In pathogenic species, these vesicles can carry molecules which exert an active role in virulence, like polysaccharides and proteins and RNA. Objective of our study is a first characterization of extracellular vesicles in the model black yeast Exophiala dermatitidis, a poly-extremophilic species and an emerging fungal pathogen in Europe. The fungus indeed, although mostly involved in cutaneous and subcutaneous mycosis and deep neurotropic infections in Asia, is more and more present in clinical reports in the West, especially in those regarding cystic fibrosis patients. We present here a study of the vesicles of $E$. dermatitidis (CBS 525.76), isolated in Japan from a male patient with chromoblastomycosis.

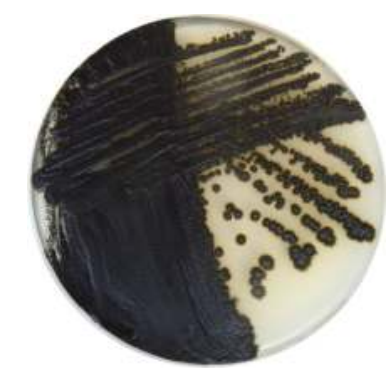

Methods. The fungus has been grown in liquid culture of $2 \% \mathrm{MEA}$ for one week at $37^{\circ} \mathrm{C}$. The vesicles have been then isolated with the ultracentrifugation method according to da Silva et al. [1] with some modifications. The culture was first centrifuged to remove the biomass, the supernatant was sterile filtrated centrifuged to remove cellular debris and finally ultra-centrifuged to obtain the vesicular fraction. The EVs have been analyzed by mean of transmission electron microscopy (TEM). Moreover, RNA and proteins have been isolated with the kit Total Exosome RNA \& Protein Isolation Kit (ThermoFisher) and the RNA sequenced with the lon Torrent technology on PGM. Proteins have been identified following LC-ESI-MS analysis of peptides. To test the vesicles for their virulence effect, we injected them into the larvae of the model host Galleria mellonella (honeycomb moth) which after $24 \mathrm{~h}$ were infected with $1 \times 10^{7}$ yeast cells/larva. The experiment lasted 7 days at the temperature of $30^{\circ} \mathrm{C}$.

TEM analysis and protein cargo of EVs. TEM analysis reveals that the vesicles of $E$. dermatitidis have a quite homogenous size, ranging between 30 and $80 \mathrm{~nm}$ (Figure 1) and that they strongly pigmented, with melanin mainly present on the outer membrane. This was confirmed by the results from the Nanoparticle Tracking analyzer (Zetaview $\left.{ }^{\circledR}\right)$ (data not shown).

The identified vesicular proteins mainly belong to the following categories:

- Cell wall proteins [Endo-glucanase;GPI anchored cell wall beta];

Oxidoreductases [aryl-alchool and choline dehydrogenase]

- Fungal virulence factor [laccase, 3-phytase, aspergillopepsin I];

- Uncharacterized protein.

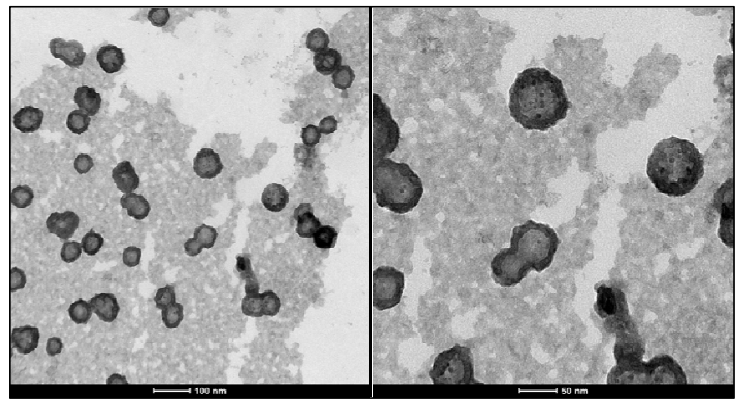

Fig.1 E. dermatitidis EVs visualized by TEM.

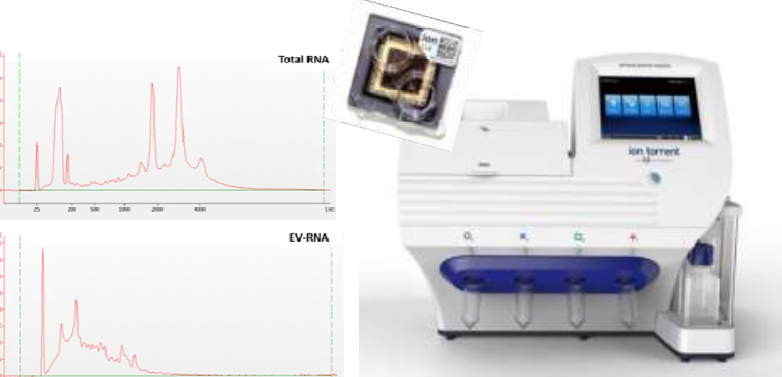

Vesicular RNA population. EVs RNA-Seq revealed the presence of tRNAs, spliceosomal RNA and small nucleolar RNA. Among the latter, snoR38.1 and SNORD14 were also isolated in E. dermatitidis during exvivo skin infection and in $T$. rubrum, also a dermatophyte fungus [2,3]. The predicted structure of SNORD14 shows that it could be processed by DICER and function as small interfering RNA (siRNA). Target genes are mostly related to neuron-development/specific proteins [AZIN1, Nedd8, PLXNA1, RAP2A] and Human Keratin [KRT40], which well fit with the pathogenesis of $E$. dermatitidis.

Fig.2 Left: Total RNA and EVs-RNA pattern. Right: PGM Sequencer

Virulence tests with G. mellonella. The injection of $E$. dermatitidis EVs into the host resulted in a rapid killing of the larvae within $24 \mathrm{~h}$, while the fungus infection led to a total larvae mortality in longer time (Fig. 3).

The injection of $E$. dermatitidis EVs into the larvae of $G$. mellonella before infection with the yeast itself decreased the host survival (data not shown), suggesting that, differently from what observed in C. albicans, the EVs of $E$. dermatitidis do not trigger a protective host response.
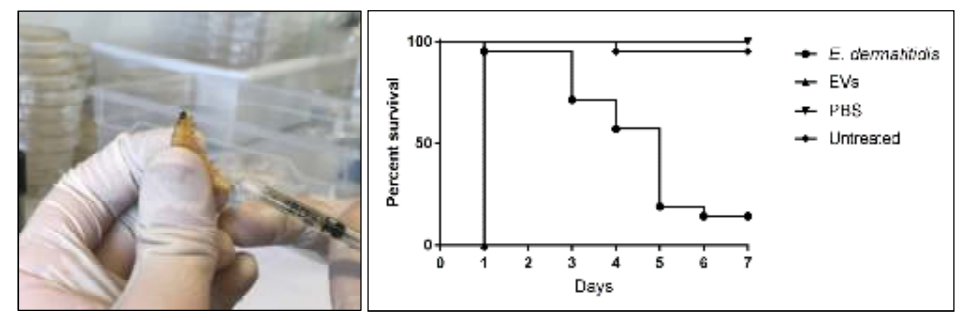

Fig.3 Left: G. mellonella injection with EVs. Right: Kaplan-Meier curve of survival

In conclusion, this first characterization of $E$. dermatitidis extracellular vesicles opens new perspectives to the comprehension of the pathobiology of the black yeasts. The molecular cargo of the vesicles gives a hint of their role in fungal virulence and it is currently investigated in human fibroblast and keratinocytes. Moreover, the vesicles seems to have a role in fungal virulence in the model host $G$. mellonella, as already observed in C. albicans and further experiment on the dose-dependent effect are currently being performed. The function of EVs needs to be further investigated in other experimental models, such as human cell lines that mimic pathogenic scenarios of the fungus. 